\title{
BMJ Open Quality Framework for patient, family-centred care within an Australian Community Hospital: development and description
}

\author{
Thuy Frakking (D , ${ }^{1,2}$ Suzanne Michaels, ${ }^{3}$ Jane Orbell-Smith, ${ }^{4}$ Lance Le Ray ${ }^{5}$
}

To cite: Frakking T, Michaels S, Orbell-Smith J, et al. Framework for patient, family-centred care within an Australian Community Hospital: development and description. BMJ Open Quality 2020;9:e000823. doi:10.1136/ bmjoq-2019-000823

Received 4 September 2019 Revised 31 March 2020 Accepted 18 April 2020
Check for updates

(C) Author(s) (or their employer(s)) 2020. Re-use permitted under CC BY-NC. No commercial re-use. See rights and permissions. Published by BMJ.

${ }^{1}$ Research Development Unit, Metro North Hospital and Health Service, Herston, Queensland, Australia

${ }^{2}$ School of Health and Rehabilitation Sciences, The University of Queensland, Saint Lucia, Queensland, Australia

${ }^{3}$ Engagement \& Integration, Caboolture Hospital, Metro North Hospital and Health Service, Caboolture, Queensland, Australia

${ }^{4}$ Education \& Training, Caboolture Hospital, Metro North Hospital and Health Service, Caboolture, Queensland, Australia

${ }^{5}$ Executive Management, Caboolture Hospital, Metro North Hospital and Health Service, Caboolture, Queensland, Australia

Correspondence to Dr Thuy Frakking;

thuy.frakking@health.qld.gov.au

\section{ABSTRACT}

Objective To describe the development of a patient and family-centred care (PFCC) conceptual framework within a small community Australian Hospital.

Methods A scoping review of scientific and grey literature and community hospital stakeholder discussions were used to identify and design a conceptual framework for PFCC across five core pillars of leadership, engagement, service delivery, learning and environment.

Results 107 publications were identified and 76 were included for data extraction. A draft framework was constructed and modified following consultation with hospital stakeholders across a small Australian Community Hospital. The 'Caring Together' framework outlines three core layers: (1) the focus of our care is the experiences of our consumers and staff; (2) concepts of leadership, environment, service delivery, engagement and learning; and (3) the overarching fundamental values of being heard respected, valued and supported by staff and consumers at all levels in an organisation.

Conclusions The conceptual Caring Together framework structures key PFCC concepts across organisational priority areas within an Australian healthcare setting and can be used to guide implementation of PFCC at other small hospital facilities. Changes to national and state healthcare funding may help facilitate improved hospital facility implementation of PFCC, and ultimately improve consumer healthcare satisfaction and clinical outcomes.

\section{BACKGROUND}

Patient, family-centred care (PFCC) is holistic care which acknowledges that patients, families and staff are central to the delivery of better healthcare. ${ }^{1}$ In an ideal healthcare system, patients and families should be empowered to be an equal and active player in their healthcare via collaborations with healthcare professionals and the healthcare system. The utilisation of various aspects of PFCC have led to improved health economics and more efficient use of healthcare services ${ }^{23}$ and improved quality of healthcare, relationships and consumer and staff experiences for patients with dementia, ${ }^{4-6}$ cancer, ${ }^{7-9}$ frail elderly, ${ }^{10}{ }^{11}$ cardiac $^{12-15}$ and diabetes. ${ }^{16}{ }^{17}$ Despite emerging literature on the positive effects of PFCC in healthcare, little is known on how to practically embed PFCC broadly across a community hospital setting which covers the care continuum from paediatrics to the frail elderly population within acute and ambulatory care settings.

In Australia, there have been national health reform initiatives acknowledging the importance of PFCC. This includes: (1) the Australian Charter of Healthcare Rights, ${ }^{18}$ which ensures consumers and providers have a shared understanding of individual's rights to accessing healthcare; (2) the Australian Safety and Quality Framework for Health Care, ${ }^{19}$ which highlights consumer centred care as one of the core pillars for safe and high quality care; and (3) mandatory accreditation for all hospitals using the National Safety and Quality Health Standard, which also recognises the collaborative value of consumers and health professionals in the delivery of healthcare..$^{20}$ Consistent with national reforms, legislation in Queensland related to the Hospital and Health Boards Act $2011^{21}$ changed governance from a Central Health Service District to Local Hospital \& Health Networks in July 2012. This change in legislation ensures local communities and consumers determine their individual healthcare service needs.

Given the emerging literature on the benefits of PFCC in healthcare settings and government legislation and policies to support PFCC, there are limited evidencebased frameworks available to support how PFCC is embedded in small Australian hospitals. Our hospital is a 265-bed facility (Level 4, Clinical Services Capability Framework) ${ }^{22}$ and provides adult and paediatric services across acute and ambulatory care settings to a socioeconomically disadvantaged population. Current international PFCC institutes, such as Planetree, ${ }^{23}$ provide an accreditation process; however, costs to access and become PFCC accredited are typically out of reach for community hospital budgets. State frameworks such as the Australian Capital Territory Health's Consumer Feedback 
Management Policy, ${ }^{24}$ New South Wales Health Caring Together: Health Action Plan, ${ }^{25}$ Western Australia Health Consumer, Carer and Community Engagement Framework, ${ }^{26}$ and Health Consumers Queensland and The Consumer and Community Engagement Framework, ${ }^{27}$ all provide general guidance on consumer engagement and feedback but are limited in evidence underpinning their development and implementation. Thus, the aim of this study was to develop an evidence-based conceptual framework that can be used to implement PFCC practices across all aspects of a small community Australian Hospital.

\section{METHODS}

In 2015, an independent staff and consumer survey $(\mathrm{n}=500)$ identified that PFCC was a key area for improvement at our small Australian Community Hospital. As a result, a scoping review of scientific and grey literature; and community, staff and consumer engagement processes were used to identify and design a conceptual framework for PFCC within our healthcare setting. The scoping review was undertaken as a quick and accurate method of identifying relevant information sources while ensuring search results are broad enough to capture wider discussion and findings. ${ }^{28}$ Discussions with stakeholders across our facility were used to complement findings from the literature and ensured concepts proposed in the conceptual framework were recognised, understood and supported for future implementation.

\section{Scoping review}

Initially, a scientific literature search was undertaken by the lead author. In January 2019, a comprehensive search strategy was developed in consultation with a professional health librarian to identify and review topical publishing in the electronic databases: Cochrane, Medline, Embase, SocIndex, Cummulative Index to Nursing and Allied Health Literature (CINAHL) and AustHealth. References cited in retrieved publications were also scanned for inclusion. Retrieved articles discussed patient care models and associated aspects including value, service delivery, engagement, learning, environment and staff/ patient experiences. The following inclusion and exclusion criteria were applied:

- Inclusion criteria: model (eg, framework or theory) or key elements of patient-centred care, attitude of health personnel, empathy, hospitals and community.

- Exclusion criteria: etters to the editor, editorials, commentaries and non-English language.

Reviewing consisted of a two-step process, review of the title, abstract and keywords, and thereafter review of the full text. A search of the grey literature was also undertaken. This identified resources including relevant topic information produced by government and not-for-profit health organisations.

The scientific literature and comprehensive literature search yielded 107 unique publications. After reviewing titles and abstracts, 85 publications remained. After fulltext reviewing, 76 articles were included in this study for contribution towards the conceptual framework for PFCC implementation within our healthcare facility. A majority of the articles included in this study were descriptive studies and included a heterogeneous sample of adult and paediatric clinical populations within healthcare settings worldwide.

The search for relevant grey literature led to the inclusion of Australian government websites, frameworks and guidelines which were relevant to PFCG practices. This includes The Australian Institute for Patient and Family Centred Care, ${ }^{1}$ Australian Charter of Healthcare Rights, ${ }^{18}$ The Australian Safety and Quality Framework for Health Care ${ }^{19}$ National Safety and Quality Health Service Standards, ${ }^{20}$ Queensland Hospital and Health Boards Act 2011, ${ }^{21}$ Metro North Hospital and Health Service Clinical Services Capability Framework Self-Assessment, ${ }^{22}$ Planetree, ${ }^{23}$ Australia Capital Territory Health's Consumer Feedback Standards, ${ }^{24}$ Caring Together: The Health Action Plan for New South Wales, ${ }^{25}$ Western Australia Health Consumer, Carer and Community Engagement Framework, ${ }^{26}$ Consumer and Community Engagement Framework for Health Organisations and Consumers, ${ }^{27}$ New South Wales Clinical Excellence CommissionGuide to Health Literacy ${ }^{29}$ and Australian Commission on Safety and Quality in Health Care-Health Literacy ${ }^{30}$ and One View Health Care PFCG. ${ }^{31}$

\section{Community, staff and consumer engagement processes}

Between October and December 2015, multiple focus groups were held to ascertain what PFCC meant to clinicians (eg, medicine, nursing, allied health), non-clinical staff (eg, administration, service improvement, research), community organisations (eg, general practitioners, local schools) and consumers. Key themes expressed in each focus group were collated and combined with targeted grey literature into a conceptual framework.

The draft conceptual framework was developed by the authors based on a combination of the results of the scoping review and key themes expressed in the community, staff and consumer focus groups. The draft conceptual framework was subsequently presented at facility-level executive and PFCG governance committees for feedback and endorsement. Our facility executive and PFCG governance committees are made up of representatives from: consumers (eg, past patients, health consumer representatives); clinicians and executives across medicine, nursing, allied health, administration, finance and communications. A revised conceptual framework was then adapted and expanded on based on feedback received. Further adaptations were made which aligned with relevant information from our hospital and health service wide key strategic priorities (eg, strategic plan 2014-2018; health services strategy 2015-2020; customer and consumer engagement strategy 2013-2015; clinician engagement strategy 2012-2015) and WHO key components of a well-functioning health system. ${ }^{32}$ 


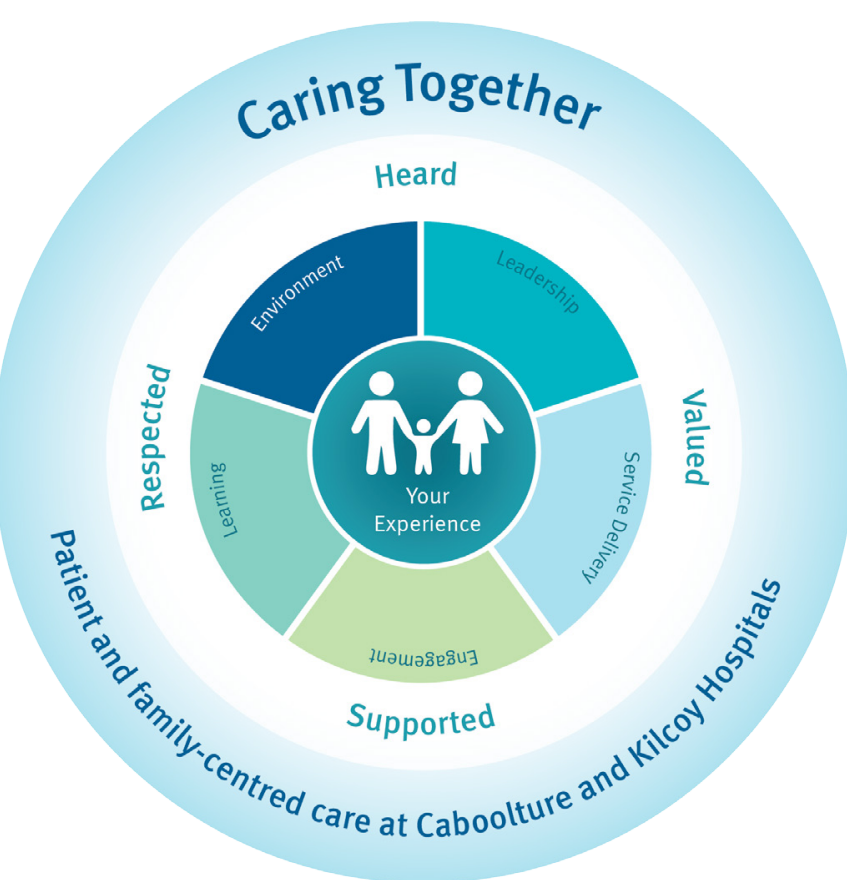

Figure 1 'Caring Together' framework to guide implementation of patient-centred care at an Australian Community Hospital.

\section{RESULTS}

\section{Caring together framework for PFCC}

Our 'Caring Together' framework (figure 1) outlines three core layers essential in embedding PFCG at our hospital facility. The innermost core reflects the experiences of our consumers and staff, considered the centre and focus of our care. The middle core layer describes four key elements of: leadership, environment, service delivery, engagement and learning. Finally, the outer core outlines the overarching fundamental values of being heard, respected, valued and supported by staff and consumers at all levels of in our organisation. The key elements integral to how we are embedding PFCC are described next.

\section{Leadership}

Changing workplace culture is challenging and that commitment from all levels of leadership within an organisation is required. ${ }^{33}$ Leadership behaviour has been positively correlated with PFCC in aged care staff $(n=3661)^{34}$ and reduced stress in staff in the aged care setting. ${ }^{35}$ Transformational leadership focuses on empowering teams and is based on the premise of mutual appreciation and reciprocal respect to achieve common goals and visions. ${ }^{36-38}$ This type of leadership style has been associated with better team performance, patient care and staff well-being and satisfaction. ${ }^{39} 40$ High-level participation from nursing staff in organisational PFCC initiatives is more likely when strengths-based transformational leadership is used within a healthcare facility. ${ }^{41}$

Examples of transformational leadership under our Caring Together framework include governance changes that incorporate the values of PFCC into our organisation's vision and code of conduct; and including a dedicated position within our organisational structure focused on transforming workplace culture towards PFCC. Patients, their families and the community are involved in the governance of our organisation. This involves including consumer representatives as equal members in governing committees and establishing a PFCC governing body to operate and meet on a regular basis to support and encourage PFCC initiatives. Leaders within our organisation model PFCC by interacting and engaging with front-line staff, patients and families; and/or consumer representatives. Ideas and feedback are welcomed and actioned via monthly staff forums, communication with our executive director via direct email, and consumer feedback forms located in all clinical areas. In addition, it is mandatory that the recruitment of all senior medical and executive management positions at our facility include at least one consumer representative on interview panels.

\section{Engagement}

Engagement is a core pillar in our PFCC Caring Together framework because high-quality patient-practitioner communication can lead to improved healthcare experiences and minimise unnecessary intervention and/or anxiety for patients, ${ }^{42}$ particularly in the area of cancer care $^{8}$ and in pharmacy practice. ${ }^{43}$ Engagement is defined as a complex interplay between practitioner and consumer relationship, which is constantly evolving and mutually contributed towards and based on each other's actions; and perceptions of each other's actions. ${ }^{44}$ Engagement from all staff within our organisation is fundamental to the relationships built with our patients and their family relatives for the effective delivery of our healthcare services and outcomes. The way healthcare staff respond to patients can influence the patient's perceptions of positive and negative experiences. ${ }^{45}$ Verbal and non-verbal communication strategies that validate and acknowledge other's emotions and perspectives are preferred within our PFCC framework, as this leads to more positive benefits to the patient-practitioner relationship in a variety of healthcare settings. ${ }^{46}$

A communication approach which acknowledges the patients personal journey, their individual personality, preferences, current abilities and ongoing need for support, is recommended to support individualised care and the patient's sense of autonomy and control with their healthcare journey. ${ }^{10} 4748$ Studies in paediatrics, ${ }^{49}$ adult diabetes, ${ }^{16}$ internal medicine and primary care ${ }^{50}$ have also reported similar results in relation to consumer preferences on individualised engagement and a neutral communication style. ${ }^{51} 52$ In our organisation, mandatory training is provided to all staff to ensure meaningful interactions and professional communication styles. ${ }^{53}$ Onboarding of new medical staff includes a 1-hour educational session of our PFCC values and principles, which is jointly delivered by hospital staff and consumers. Specific training provided to new staff include: (1) 
communication, respect, accountability equals safe healthcare training course; (2) probe, alert, challenge, emergency language; and situation, background, assessment, recommendation tools.

Staff engagement is also important under the engagement element of our Caring Together framework. Improved job satisfaction has been shown when staff perceptions of PFCC in their unit is high, compared with other units with less focus on PFCC. ${ }^{54}$ Improvements in job satisfaction, staff retention, staff well-being and better quality patient care have resulted when leaders empower their staff to act in ways that are fitting and that they are left to trust making their own decisions, particularly in nursing homes. ${ }^{37}$ Staff also need to be orientated and managed in a PFCC way for a PFCC workplace culture to be possible. ${ }^{37556}$ Bright $e t$ a $t^{44}$ found that engaged practitioners (eg, staff who showed passion, laughter, positive facial expression) improved patient outcomes and patient satisfaction. Conversely, disengaged practitioners impacted on the patient's confidence (eg, feelings of isolation) and how they delivered services (eg, delay in offering interventions).

Examples of staff engagement under our PFCC Caring Together framework include a committee for planning of staff events; annual staff health and well-being themed programmes; quarterly barbeques to thank staff for their ongoing commitment to the organisation; and staff are kept informed of organisational updates via a monthly staff forum and a 'Caring Together in Action' staff newsletter. In our organisation, we celebrate and acknowledge staff under the 'Shining Star' and 'Heart Felt' categories on a monthly basis. The Shining Star award is peer nominated in recognition of small actions which help the work of others while the Heart Felt award is patient nominated for outstanding service. We also host an annual 'Caring Together Excellence Awards', which celebrate staff in varying categories such as 'values in action'. In addition, systems are in place to support professional development and career advancement such as annual professional development plans for all staff within the organisation. Our staff also have access to local gym and fitness clubs via our 'Fitness Passport' programme-a joint initiative between our community and hospital facilities.

\section{Service delivery}

Service delivery under our PFCC Caring Together framework strives to facilitate the best possible care experiences through shared decision-making processes and integration of care. There is increasing evidence that the inclusion of caregiver/family involvement in healthcare planning, also known as shared decision-making, can minimise adverse events and complications ${ }^{57-62}$ Further, caregivers prefer preventative supports over reactionary supports to minimise burnout and other detrimental health outcomes. ${ }^{63}$ In support of shared decision-making in healthcare ${ }^{64}$ studies on adults with cardiac issues have demonstrated that significantly improved self-efficacy and return to preactivity levels occur when patients are allowed to share their story; and share in the development and ownership of their own healthcare management plan. ${ }^{12} 13$

In our organisation, shared decision-making processes can be a passive or active process for the patient, depending on their preferences. To empower and assist our patients and families to actively participate in their care at our hospital, we have 'speak up, ask questions' posters displayed on walls in all clinical areas. For our admitted inpatients, the provision of patient discharge envelopes (known as patient powerpacks) is provided to patients and their families. The patient powerpack lists information which helps prompts our patients and their families to ask specific questions while in hospital and provides a canvass for our staff to place and discuss relevant care information in for appropriate follow-up post discharge. ${ }^{656}$ For caregivers who choose to stay overnight, our organisation has a boarding policy which allows caregivers appropriate access to food and a temporary bed. Finally, the incorporation of a consumer living with dementia to help staff and management codesign our newly opened 'Gentlemen and Ladies Ageing with Dignity' unit is another example of how our facility attempts to use shared-decision making processes in major organisational planning.

Integrated healthcare for patients with multiple comorbidities requires flexibility as the needs of consumers change over time and adapting to those needs promotes PFCC. ${ }^{16} 6768$ Service access across health and social care sectors is important at our facility, given our low socioeconomic demographics. ${ }^{67}$ Under PFCC, service partners need to work together to minimise limitations which impact on service access for consumers such as resource (eg, waitlist times) and location (eg, distance to appropriate service) barriers. ${ }^{11} 69$ Improved health-related outcomes, patient satisfaction and reduced healthcare system costs have been shown when coordination of care for adults and children with chronic disease is managed in a coordinated way. ${ }^{70-73}$ Integration of care based on our PFCC Caring Together framework highlights that strategies are multifaceted and can include health promotion, care coordination; service partnerships; and multidisciplinary collaboration. ${ }^{58} 6773-77$

Specific examples of integrated care at our hospital facility include: partnering with general practices for service access planning (eg, team care arrangements); multiple nurse navigator positions; daily multidisciplinary team meetings to facilitate discharge planning for inpatients; use of telehealth to support access to care when patients are located in rural regions; formal research into healthcare pathways for children with developmental chronic conditions; and involvement in community healthcare alliances comprising local schools, government, non-government and private community agencies.

\section{Learning}

Continual PFCC education and learning from staff and consumer experiences are essential in improving the 


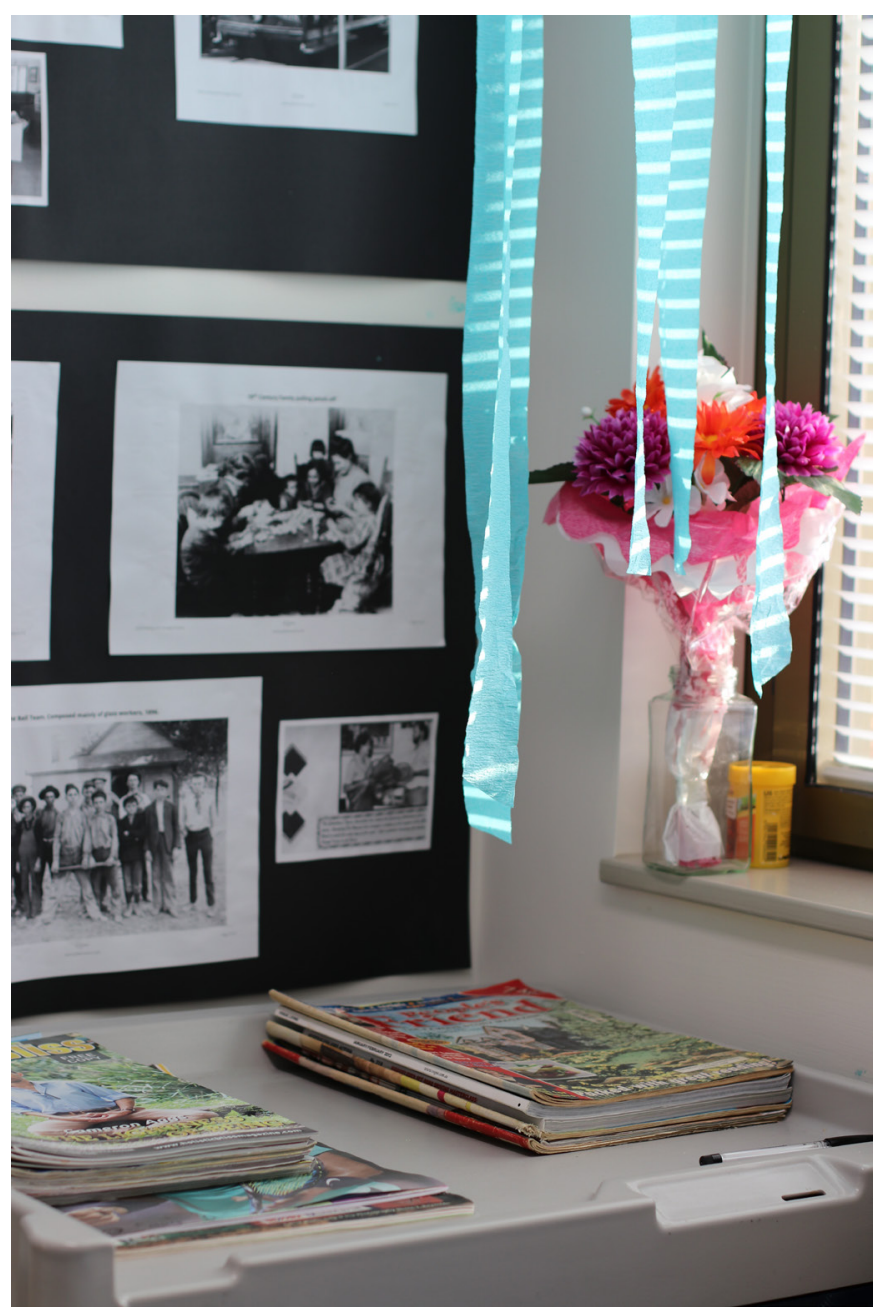

Figure 2 Patient, family-centred care environmental modification of social activity area located on ward.

design and delivery of healthcare services. ${ }^{78}$ Increased knowledge in PFCC and acceptance of PFCC patientpractitioner relationships is possible when a 4-day training course on PFCC is provided to a group of allied health professionals. ${ }^{79}$ At our facility, mandatory orientation for all new staff is provided and includes an informational session on our PFCC Caring Together mission, values and framework. All staff in our organisation are also required to complete annual cultural awareness training. Regular PFCC training is tailored and provided to staff based on consumer and staff feedback via our compliments and complaints management system.

Our facility also recognises that low health literacy in our population influences on how we discuss and provide education, intervention and follow-up care options for patients. Health literacy is defined as a person's ability to understand information, resources, supports and environments available to them to manage their healthcare. Low health literacy has been attributed with poorer health outcomes, reduced compliance to healthcare plans, increased neonatal morbidity and increased healthcare costs. ${ }^{80}$ Specific strategies to increase health literacy include: training health literacy 'champions' throughout the organisation; consumers reviewing patient education resources; using plain language (ie, use of short sentences and an active voice); checking the patient's understanding using the 'teach-back' method; and having patient educational resources which have plenty of white space. ${ }^{82}$ In Australia, there are practical guides available on the implementation of health literacy friendly facilities, including guidelines by the New South Wales Clinical Excellence Commission ${ }^{29}$ and Australian Commission on Safety and Quality in Health Care. ${ }^{30}$

\section{Environment}

Comfort is embedded to PFCC frameworks worldwide and acknowledged as an important element in PFCC. ${ }^{31} 83$ Comfort is multifaceted and includes patient's physical discomfort, perception of control, optimism and resilience; and the personal sense that they are being cared for and valued by healthcare staff. ${ }^{84}$ Using the 'Comfort Always Matters' framework, ${ }^{84}$ comfort for a consumer is complex and intertwined by the level of family involvement, level of staff engagement/skillset/assistance and physical factors, such as ambience, which impact on the clinical environment. Healthy workplace environments are essential in facilitating PFCC culture as positive correlations exist between healthy patients, staff well-being and healthy workplace environments. ${ }^{85}$ Strategies to promote PFCC healthcare environments focus on a safe environment and include: avoiding clutter in the hallways, increased lighting, using clear signage for patients and families to navigate the hospital, and dedicated areas for patients to have social interactions. ${ }^{86-89}$ In our facility, social activity areas have been implemented on adult wards to encourage patients to socially connect and stimulate their minds with magazines, drawings and sudoku puzzles. Each social activity area has brightly coloured decorations—see figure 2.

\section{DISCUSSION}

This article has outlined the development of our Caring Together conceptual framework used to guide how we embed PFCC within our Australian hospital facility. Many studies on PFCC to date have gathered PFCC perspectives from interviews with professional staff and consumers, but lack strong empirical methodological designs to evaluate levels of engagement and PFCC. ${ }^{90}{ }^{91}$ Future studies could consider using formal validated outcome measures on evaluating different aspects of PFCC, including the five core pillars outlined in our Caring Together framework. The Primary Health Care Engagement Scale ${ }^{92}$ and Valuing Patients as Individuals Scale ${ }^{93}$ are possible scales for consideration as emerging validated outcome measurement tools in levels of engagement and PFCC.

Although our facility endorses and supports PFCC, we acknowledge that in some clinical contexts (eg, intensive care units), ${ }^{94}$ the implementation of some components of our Caring Together framework (eg, shared decisionmaking in service delivery) may be more difficult due 
to the nature of the patient's biophysical compromise. Nevertheless, the Caring Together framework is intended as a guide only and it is important that individual clinical areas use different aspects of the framework to best promote PFCC in the most safe and efficient way for patients, families and staff. In addressing some of the limitations in implementation within the intensive care unit setting, chaplains and social workers have been identified as valuable resources in the intensive care unit as they are not only able to provide nurses with the relevant supports, but are able to meet the emotional and psychosocial needs of the patients using PFCC communication. ${ }^{95}$ In addition, introducing an intensive care unit diary for patients and families can increase PFCC and improve teamwork and communication between staff, patients and families. ${ }^{96}$ This highlights that PFCC within difficult clinical settings, such as the intensive care unit, is in fact possible.

Current models of healthcare funding for Australian public hospitals are predominately based on diagnosticrelated groups (DRG) ${ }^{97}$ It has been suggested that this type of funding model promotes under servicing to lower costed DRGs and over servicing of higher priced DRGs ${ }^{98}$ to achieve higher reimbursement. ${ }^{99}$ DRG-based hospital funding does not use comprehensive casemix data (eg, patient-related factors of multiple comorbidities, satisfaction) which are intrinsic to the effective management of health conditions. ${ }^{98}$ At present, there are no criteria for successful levels of PFCC implementation in current Australian healthcare funding models. The USA and UK are arguably considered the leaders in PFCC and evaluate consumer satisfaction within their relevant healthcare models. Consumer satisfaction, as key performance indicators for the distribution of healthcare funding, has led to increased consumer satisfaction, ${ }^{100}$ but variable sustained improvements in clinical outcome measures. ${ }^{101}$ Increased implementation of PFCC practices into Australian hospital facilities are more likely to occur when there are financial rewards and/or components of healthcare funding to motivate hospital facilities to implement PFCC. Future funding allocation models could consider a balance between DRGs, level of consumer and clinician satisfaction, and evidence-based management and innovation. As such, block budgets for hospital and health services across Australia may be an option to help allow sustainable implementation of PFCC. In addition, rigorous cost-effectiveness evaluation plans of PFCC and clinical outcomes should mandatory to support sustainability and future adaptations to proposed funding models in Australian healthcare. ${ }^{102}$

Limitations in the development of our PFCC Caring Together framework includes the use of a scoping review and completion of site-specific focus groups without defined study protocols, which impacted on our ability to definitively draw conclusions for broader generalisation of our PFCC framework to other Australian hospitals. Nevertheless, the advantage of our novel approach which combines consumer perspectives with available evidence base provides a solid foundation for the development of a PFCC framework that is specific to the needs of our small Australian community hospital. Our study has improved our understanding of PFCC practices and enabled us to identify the multiple influences on PFCG practice implementation within our hospital facility. Our PFCC Caring Together framework outlines five core pillars which have guided how we are embedding PFCC at our hospital facility. PFCC is not an initiative or a project, it is a combination of systems, procedures and behaviours that are rigid enough to provide consistent safe care, but flexible enough to meet the needs of evolving legislation, a changing workforce and diverse patient cohort. The five core pillars describe updated literature across leadership, engagement, service delivery, learning and environment within healthcare settings; and acknowledges that our patients and healthcare colleagues remain the central focus in all our day-to-day activities. The ongoing implementation of PFCC practices in our facility will be guided by future local and international research outcomes; and changes in organisational and Australian government policies.

Contributors LLR and SM conceptualised the study. TF and JO-S planned the study. All authors contributed towards data collection throughout the study. TF and JO-S extracted and reviewed all articles associated with the scoping review. TF drafted the initial manuscript. All authors provided critical intellectual input into the final manuscript.

Funding The authors have not declared a specific grant for this research from any funding agency in the public, commercial or not-for-profit sectors.

Competing interests None declared.

Patient and public involvement Health professionals and consumers participated in an online survey and focused groups. Our local PFCC governance committee, which consists of consumers, staff and executives, provided initial feedback and final endorsement of our PFCC 'Caring Together' framework. Patients were not invited to participate in the scoping review component of this study due to the technical nature of skills required.

Patient consent for publication Not required.

Provenance and peer review Not commissioned; externally peer reviewed.

Data availability statement There are no data in this work.

Open access This is an open access article distributed in accordance with the Creative Commons Attribution Non Commercial (CC BY-NC 4.0) license, which permits others to distribute, remix, adapt, build upon this work non-commercially, and license their derivative works on different terms, provided the original work is properly cited, appropriate credit is given, any changes made indicated, and the use is non-commercial. See: http://creativecommons.org/licenses/by-nc/4.0/.

ORCID iD

Thuy Frakking http://orcid.org/0000-0003-2724-6919

\section{REFERENCES}

1 Hush Foundation. Patient \& Family Centred Care [Website]. Available: https://www.hush.org.au/patient-and-family-centred-care

2 Bertakis KD, Azari R. Patient-Centered care is associated with decreased health care utilization. J Am Board Fam Med 2011;24:229-39.

3 Stone S. A retrospective evaluation of the impact of the Planetree patient-centred model of care on inpatient quality outcomes. Health Environments Res Design J 2008:59-69.

4 Barbosa A, Sousa L, Nolan M, et al. Effects of Person-Centered care approaches to dementia care on staff. Am J Alzheimers Dis Other Demen 2015;30:713-22. 
5 McMillan SS, Kendall E, Sav A, et al. Patient-Centered approaches to health care: a systematic review of randomized controlled trials. Med Care Res Rev 2013;70:567-96.

6 Rave N, Geyer M, Reeder B, et al. Radical systems change. innovative strategies to improve patient satisfaction. J Ambul Care Manage 2003;26:159-64.

7 Kane HL, Halpern MT, Squiers LB, et al. Implementing and evaluating shared decision making in oncology practice. CA Cancer J Clin 2014:64:377-88.

8 Thorne SE, Bultz BD, Baile WF, et al. Is there a cost to poor communication in cancer care?: a critical review of the literature. Psychooncology 2005;14:875-84

9 Tremblay D, Roberge D, Touati N, et al. Effects of interdisciplinary teamwork on patient-reported experience of cancer care. BMC Health Serv Res 2017;17:218.

10 Chung PYF, Ellis-Hill C, Coleman P. Supporting activity engagement by family carers at home: maintenance of agency and personhood in dementia. Int J Qual Stud Health Well-being 2017;12:1267316.

11 Threapleton DE, Chung RY, Wong SYS, et al. Integrated care for older populations and its implementation facilitators and barriers: a rapid scoping review. Int J Qual Health Care 2017;29:327-34.

12 Fors A, Ekman I, Taft C, et al. Person-Centred care after acute coronary syndrome, from hospital to primary care - a randomised controlled trial. Int J Cardiol 2015;187:693-9.

13 Fors A, Gyllensten H, Swedberg K, et al. Effectiveness of person-centred care after acute coronary syndrome in relation to educational level: subgroup analysis of a two-armed randomised controlled trial. Int J Cardiol 2016;221:957-62.

14 Jansson I, Fors A, Ekman I, et al. Documentation of person-centred health plans for patients with acute coronary syndrome. Eur $J$ Cardiovasc Nurs 2017;1474515117718415.

15 Meterko M, Wright S, Lin $\mathrm{H}$, et al. Mortality among patients with acute myocardial infarction: the influences of patient-centered care and evidence-based medicine. Health Serv Res 2010;45:1188-204.

16 Bunn F, Goodman C, Reece Jones P, et al. What works for whom in the management of diabetes in people living with dementia: a realist review. BMC Med 2017;15:141.

17 Cheng L, Sit JWH, Choi K-C, et al. Effectiveness of a patientcentred, empowerment-based intervention programme among patients with poorly controlled type 2 diabetes: a randomised controlled trial. Int J Nurs Stud 2018;79:43-51.

18 Australian Charter of Healthcare Rights. Australian Commission on safety and quality in health care. Available: https:www. safetyandquality.gov.au/national-priorities/chart-of-healthcarerights/

19 The Australian Safety and Quality Framework for Health Care [Website]. Australian Commission on Saftey and quality in health care. Available: https://www.safetyandquality.gov.au/nationalpriorities/australian-safety-and-quality-framework-for-health-care

20 National Safety and Quality Health Service Standards. Australian Commission on Safety and Quality in Health Care; [1st edition. Available: https://www.safetyandquality.gov.au/wp-content/ uploads/2011/09/NSQHS-Standards-Sept-2012/pdf

21 Queensland Government. Queensland hospital and health boards act, 2011. Available: https://www.legislation.qld.gov.au/LEGISLTN/ CURRENT/H/HHNA11.pdf

22 Clinical Services Capability Framework Self-Assessment. Metro North hospital and health service, 2015. Available: https://www. health.qld.gov.au/data/assets/pdf file/0025/634903/cscfselfassess-sum-metro-north.pdf

23 Planetree: Planetree organisation. Available: www.planetree.org

24 Act health's consumer feedback standards: act health. Available: https://www.health.act.gov.au/sites/default/files/Consumerfeedback standards-listeningandlearning.pdf

25 Caring together: the health action plan for NSW: NSW health Available: http://www.health.nsw.gov.au/publications/Pages/caringtogether-plan.aspx

26 Western Australia (WA) health consumer, carer and community engagement framework East Perth: government of Western Australia, department of health. Available: http://ww2.health.wa.gov. au/Articles/A_E/Consumer-carer-and-community-engagement

27 Consumer and community engagement framework for health organisations and consumers: Healh consumers Queensland. Available: www.hcq.org.au/wp-content/uploads/2017/03/HCQCCE-Framework-2017.pdf

28 Armstrong R, Hall BJ, Doyle J, et al. 'Scoping the scope' of a cochrane review. J Public Health 2011;33:147-50.

29 NSW Clinical Excellence Commission. Guide health literacy.

30 Australian Commission on Safety and Quality in Health Care. Health literacy: taking action to improve safety and quality, 2014.

31 The eight principles of patient-centered care 2017
32 WHO. Key components of a well functioning health system. Geneva, 2010.

33 Moore ML, Putman PA. Cultural transformation toward patient safety: one conversation at a time. Nurs Adm Q 2008;32:102-8.

34 Backman A, Sjögren K, Lindkvist M, et al. Towards personcentredness in aged care - exploring the impact of leadership. $J$ Nurs Manag 2016;24:766-74.

35 Orrung Wallin A, Jakobsson U, Edberg A-K. Job strain and stress of conscience among nurse assistants working in residential care. $J$ Nurs Manag 2015;23:368-79.

36 Avolio BJ, Gardner WL. Authentic leadership development: getting to the root of positive forms of leadership. Leadersh $Q$ 2005;16:315-38

37 Rokstad AMM, Vatne S, Engedal K, et al. The role of leadership in the implementation of person-centred care using dementia care mapping: a study in three nursing homes. J Nurs Manag 2015;23:15-26.

38 Forbes-Thompson S, Leiker T, Bleich MR. High-performing and low-performing nursing homes: a view from complexity science. Health Care Manage Rev 2007;32:341-51.

39 McGuire E, Kennerly SM. Nurse managers as transformational and transactional leaders. Nurs Econ 2006;24:179.

40 Weberg D, Leadership T. Transformational leadership and staff retention: an evidence review with implications for healthcare systems. Nurs Adm Q 2010;34:246-58.

41 Beckett P, Field J, Molloy L, et al. Practice what you preach: developing person-centred culture in inpatient mental health settings through strengths-based, transformational leadership. Issues Ment Health Nurs 2013;34:595-601.

42 Purbhoo D, Wojtak A. Patient and Family-Centred home and community care: Realizing the opportunity. Can J Nurs Leadersh 2018;31:40-51.

43 Wolters M, van Hulten R, Blom L, et al. Exploring the concept of patient centred communication for the pharmacy practice. Int $\mathrm{J}$ Clin Pharm 2017;39:1145-56.

44 Bright FA, Kayes NM, Cummins C, et al. Co-constructing engagement in stroke rehabilitation: a qualitative study exploring how practitioner engagement can influence patient engagement. Clin Rehabil 2017;31:1396-405

45 Olsson E, Ingvad B. The emotional climate of care-giving in homecare services. Health Soc Care Community 2001;9:454-63.

46 Hafskjold L, Sundling V, van Dulmen S, et al. The use of supportive communication when responding to older people's emotional distress in home care - An observational study. BMC Nurs 2017;16:24.

47 Burton CD, Entwistle VA, Elliott AM, et al. The value of different aspects of person-centred care: a series of discrete choice experiments in people with long-term conditions. BMJ Open 2017;7:e015689.

48 Al Danaf J, Chang BH, Shaear M, et al. Surfacing and addressing hospitalized patients' needs: Proactive nurse rounding as a tool. $J$ Nurs Manag 2018;26:540-7.

49 Burton KLO, Bau K, Lewis J, et al. Using family and staff experiences of a botulinum toxin-A service to improve service quality. Child Care Health Dev 2017;43:847-53.

50 Wolf A, Moore L, Lydahl D, et al. The realities of partnership in person-centred care: a qualitative interview study with patients and professionals. BMJ Open 2017;7:e016491.

51 Dwamena F, Holmes-Rovner M, Gaulden CM, et al. Interventions for providers to promote a patient-centred approach in clinical consultations. Cochrane Database Syst Rev 2012;12:CD003267.

52 Liu W, Gerdtz M, Manias E. Creating opportunities for interdisciplinary collaboration and patient-centred care: how nurses, doctors, pharmacists and patients use communication strategies when managing medications in an acute hospital setting. J Clin Nurs 2016;25:2943-57.

53 Watson B, Bugden S, Machen R, et al. Evaluation of the 'Communication and Patient Safety' Training Program 2017.

54 Rathert C, May DR. Health care work environments, employee satisfaction, and patient safety: care provider perspectives. Health Care Manage Rev 2007;32:2-11.

55 Sheard D. Bringing relationships to the heart of dementia care. J Dementia Care 2004;12:22-4.

56 Fix GM, VanDeusen Lukas C, Bolton RE, et al. Patient-Centred care is a way of doing things: how healthcare employees conceptualize patient-centred care. Health Expect 2018;21:300-7.

57 Gill FJ, Leslie GD, Marshall AP. Family initiated escalation of care for the deteriorating patient in hospital: Family centred care or just "box ticking". Australian Critical Care 2016;29:195-200. 
58 Hazzard E, Barone L, Mason M, et al. Patient-Centred dietetic care from the perspectives of older malnourished patients. J Hum Nutr Diet 2017;30:574-87.

59 Doos L, Bradley E, Rushton CA, et al. Heart failure and chronic obstructive pulmonary disease multimorbidity at hospital discharge transition: a study of patient and carer experience. Health Expect 2015;18:2401-12.

60 Cornwell $\mathrm{J}$. The point of care programme and the contribution of user-centred design to patient-centred care. Health Issues 2011:107:13-14.

61 Tobiano G, Bucknall T, Marshall A, et al. Patients' perceptions of participation in nursing care on medical wards. Scand J Caring Sci 2016;30:260-70.

62 Shields L, Zhou H, Pratt J, et al. Family-centred care for hospitalised children aged 0-12 years. Cochrane Database Syst Rev 2012;10:CD004811.

63 Giosa J, Holyoke P. Caregiving is not a disease: moving from reactive to proactive supports for family caregivers across the healthcare system. Hcq 2014;17:36-41.

64 van Dongen JJJ, Habets IGJ, Beurskens A, et al. Successful participation of patients in interprofessional team meetings: a qualitative study. Health Expectations 2017:20:724-33.

65 Greysen SR, Harrison JD, Kripalani S, et al. Understanding patientcentred readmission factors: a multi-site, mixed-methods study. BMJ Qual Saf 2017;26:33-41.

66 Leino-Kilpi H, Gröndahl W, Pekonen A, et al. Knowledge received by hospital patients-a factor connected with the patient-centred quality of nursing care. Int J Nurs Pract 2015;21:689-98.

67 Leijten FRM, Struckmann V, van Ginneken E, et al. The SELFIE framework for integrated care for multi-morbidity: development and description. Health Policy 2018;122:12-22.

68 Evans J, Matheson G, Buchman S, et al. Integrating cancer care beyond the hospital and across the cancer pathway: a patientcentred approach. Healthcare Quarterly 2014;17:28-32.

69 Kirby SE, Dennis SM, Bazeley P, et al. What distinguishes clinicians who better support patients for chronic disease self-management? Aust J Prim Health 2012;18:220-7.

70 Cohen E, Lacombe-Duncan A, Spalding K, et al. Integrated complex care coordination for children with medical complexity: a mixed-methods evaluation of tertiary care-community collaboration. BMC Health Serv Res 2012;12:366-77.

71 Homer CJ, Klatka K, Romm D, et al. A review of the evidence for the medical home for children with special health care needs. Pediatrics 2008;122:e922-37.

72 Jackson GL, Powers BJ, Chatterjee R, et al. The patient-centered medical home. Ann Intern Med 2013;158:169-78.

73 Bodenheime T, Bodenheimer EH, Grumbach K. Improving primary care for patients with chronic illness: the chronic care model Part 2. JAMA 2002;288:1909-14.

74 Haigh C, Ormandy P. Evaluation of the organisation and delivery of patient-centred acute nursing care. Contemp Nurse 2011;37:253-64.

75 Li J, Talari P, Kelly A, et al. Interprofessional teamwork innovation model (ITIM) to promote communication and patient-centred, coordinated care. BMJ Qual Saf 2018;27:700-9.

76 Jowsey T, Yen L, Wells R, et al. National health and hospital reform Commission final report and patient-centred suggestions for reform. Aust J Prim Health 2011:17:162-8.

77 Paynter H, Fodero L, Scuteri J, et al. Evaluation of the McGrath Foundation's breast cancer nurses initiative. Aus J Can Nurs 2013;14:4-9.

78 Chaboyer W, McMurray A, Marshall A, et al. Patient engagement in clinical communication: an exploratory study. Scand J Caring Sci 2016;30:565-73.

79 Lawford BJ, Delany C, Bennell KL, et al. Training physical therapists in Person-Centered practice for people with osteoarthritis: a qualitative case study. Arthritis Care Res 2018;70:558-70

80 Davis TC, Williams MV, Marin E, et al. Health literacy and cancer communication. CA Cancer J Clin 2002;52:134-49.
81 Hart TL, Blacker S, Panjwani A, et al. Development of multimedia informational tools for breast cancer patients with low levels of health literacy. Patient Educ Couns 2015;98:370-7.

82 Simmons RA, Cosgrove SC, Romney MC, et al. Health literacy: cancer prevention strategies for early adults. Am J Prev Med 2017;53:S73-7.

83 Sjögren K, Lindkvist M, Sandman P-O, et al. Organisational and environmental characteristics of residential aged care units providing highly person-centred care: a cross sectional study. BMC Nurs 2017;16:44.

84 Wensley C, Botti M, McKillop A, et al. A framework of comfort for practice: an integrative review identifying the multiple influences on patients' experience of comfort in healthcare settings. Int J Qual Health Care 2017;29:151-62.

85 Your work health: whether patient or healthcare provider, a health workplace is the key to well-being. Registered Nurse $J$ 2003;15:16-19.

86 Digby R, Bloomer MJ. People with dementia and the hospital environment: the view of patients and family carers. Int $\mathrm{J}$ Older People Nurs 2014;9:34-43.

87 Waller S, Masterson A, Evans SC. The development of environmental assessment tools to support the creation of dementia friendly care environments: innovative practice. Dementia 2017;16:226-32.

88 Hung L, Phinney A, Chaudhury $\mathrm{H}$, et al. "Little things matter!" Exploring the perspectives of patients with dementia about the hospital environment. Int J Older People Nurs 2017;12:e12153.

89 Bardach NS, Lyndon A, Asteria-Peñaloza R, et al. From the closest observers of patient care: a thematic analysis of online narrative reviews of hospitals. BMJ Qual Saf 2016;25:889-97.

90 Domecq JP, Prutsky G, Elraiyah T, et al. Patient engagement in research: a systematic review. BMC Health Serv Res 2014;14:89.

91 Esmail L, Moore E, Rein A. Evaluating patient and stakeholder engagement in research: moving from theory to practice. J Comp Eff Res 2015;4:133-45

92 Kosteniuk JG, Stewart NJ, Karunanayake CP, et al. Exploratory factor analysis and reliability of the primary health care engagement (PHCE) scale in rural and remote nurses: findings from a national survey. Prim Health Care Res Dev 2017;18:608-22.

93 Jones MC, Williams B, Rattray J, et al. Extending the assessment of patient-centredness in health care: development of the updated Valuing patients as individuals scale using exploratory factor analysis. J Clin Nurs 2018;27:65-76.

94 Jakimowicz S, Perry L, Lewis J. An integrative review of supports, facilitators and barriers to patient-centred nursing in the intensive care unit. J Clin Nurs 2017;26:4153-71.

95 Gélinas C, Fillion L, Robitaille M-A, et al. Stressors experienced by nurses providing end-of-life palliative care in the intensive care unit. Can J Nurs Res 2012;44:1.

96 Blair KTA, Eccleston SD, Binder HM, et al. Improving the patient experience by implementing an ICU diary for those at risk of Postintensive care syndrome. Journal of Patient Experience 2017;4:4-9.

97 Palmer KS, Agoritsas T, Martin D, et al. Activity-Based funding of hospitals and its impact on mortality, readmission, discharge destination, severity of illness, and volume of care: a systematic review and meta-analysis. PLoS One 2014:9:e109975.

98 Bystrov V, Staszewska-Bystrova A, Rutkowski D, et al. Effects of DRG-based Hospital payment in Poland on treatment of patients with stroke. Health Policy 2015;119:1119-25.

99 Doran T, Maurer KA, Ryan AM. Impact of provider incentives on quality and value of health care. Annu Rev Public Health 2017:38:449-65.

100 Fenton JJ, Jerant AF, Bertakis KD, et al. The cost of satisfaction: a national study of patient satisfaction, health care utilization, expenditures, and mortality. Arch Intern Med 2012;172:405-11.

101 Mandavia R, Mehta N, Schilder A, et al. Effectiveness of UK provider financial incentives on quality of care: a systematic review. Br J Gen Pract 2017;67:e802:e800-15.

102 Meacock R, Kristensen SR, Sutton M. The cost-effectiveness of using financial incentives to improve provider quality: a framework and application. Health Econ 2014;23:1-13. 